\title{
Elasticidade Vertical de Memória em Docker Containers*
}

\author{
Daniel M. B. Sodré, José Victor de P. e Silva, Cristina Boeres, Vinod E. F. Rebello \\ ${ }^{1}$ Instituto de Computação - Universidade Federal Fluminense (UFF) \\ Niterói - RJ - Brazil \\ \{danielbougleux, josevictorsilva\}eid.uff.br, \{boeres, vinod\}@ic.uff.br
}

\begin{abstract}
Resumo. Nuvens computacionais executam as requisições de seus clientes em um ambiente virtual configurado de acordo com as necessidades das aplicações. O cliente normalmente paga por uma quantidade de recursos estimada para sua aplicação executar, mas esta pode vir a consumir mais ou menos recursos do que o esperado. Para evitar tais situações, o gerenciamento eficiente de recursos é essencial. Esse trabalho visa elucidar alguns pontos estudados sobre o consumo de recursos de aplicações executando em Docker containers.
\end{abstract}

\section{Introdução}

Plataformas de computação em nuvem visam uma melhor utilização de seus recursos para poder melhor atender os seus clientes. É comum, no entanto, encontrar aplicações executando na nuvem com subutilização dos recursos alocados. Neste caso, tais recursos poderiam ser associados a outras aplicações. Por outro lado, pode ocorrer o cenário oposto, onde a aplicação consome mais recursos do que o estimado, o que pode levar a uma degradação de seu desempenho ou até mesmo o término precoce.

É interessante, então, que provedores de serviços em nuvem sejam capazes de escalar os recursos da nuvem para atender a uma variação na demanda de maneira automática - essa habilidade chama-se elasticidade de recursos. Há dois tipos de elasticidade: elasticidade vertical, onde o sistema é capaz de prover mais ou menos recursos computacionais baseado na demanda das aplicações em uma instância computacional; e elasticidade horizontal, onde o sistema é capaz de aumentar ou diminuir suas instâncias computacionais para atender a variações na demanda.

\section{O Vertical Elasticity Management of Containers (VEMoC)} [Nicodemus et al. 2020] através da análise e previsão do consumo de memória das aplicações, implementa elasticidade vertical de memória em conjunto com políticas de preempção dos contêineres levando a resultados promissores tanto para o provedor quanto para os seus clientes. Considerando que os mecanismos do VEMoC utilizam LXC [Canonical Ltd. 2020], este trabalho tem por objetivo estender o framework para outras tecnologias de conteineirização como Docker[Docker Inc. 2020], uma plataforma bastante utilizada. Experimentos iniciais do Docker apresentaram certa sobrecarga de monitoramento e suspensão de contêineres. Por conta disso, uma análise mais aprofundada de alternativas para monitoramento de contêineres e mecanismos de checkpoint se faz necessária, para integrar Docker ao VEMoC.

*Este trabalho foi apoiado pelo projeto REMATCH (Processo n $\left.{ }^{\circ} 88887.310261 / 2018-00\right)$ do Programa Institucional de Internacionalização (PrInt) da CAPES e os alunos Daniel M. B. Sodré e José Victor de P. e Silva por bolsas de Iniciação Científica de CNPq/PIBIC 2020-21. 
No caminho deste aprofundamento, este trabalho aqui apresentado mostra uma análise inicial dos benefícios e desafios de gerenciadores de recursos na nuvem para aplicações executadas em Docker contêineres. Docker conta com uma implementação do Kernel Linux chamada control groups para gerenciar os recursos do contêiner, permitindo o monitoramento e a elasticidade dos recursos em ambientes Docker.

\section{Elasticidade Vertical em Docker}

Pode-se apontar certas diferenças na implementação de elasticidade vertical entre contêineres e Máquinas Virtuais (MVs). Os contêineres trabalham com limitação de recursos, que contrasta com a alocação efetiva dos recursos no caso das MVs. Assim, ao gerenciar o consumo de recursos de contêineres, a quantidade de recursos efetivamente alocada pelas aplicações dentro do contêiner em um determinado momento pode ser menor ou igual ao dado limite. A consequência disso é que, aquela quantidade de recursos não alocada em um determinado momento pode ser utilizada para outros processos/contêineres executados na mesma máquina. Desta forma, pode haver uma certa interferência entre recursos alocados a diferentes contêineres/processos. Assim, para gerenciar efetivamente utilização de recursos da nuvem, um middleware de gerenciamento deve ser capaz de manipular dinamicamente os limites de utilização de recursos dos contêineres em tempo de execução e consequentemente, melhorar o aproveitamento dos mesmos, sem causar interferência maléfica entre eles - esse é um dos conceitos implementados no VE$\mathrm{MoC}$, adicionado à manipulação de eventos de preempção de contêineres para um melhor desempenho do ambiente virtual.

Seja uma aplicação submetida, onde o provedor configura o limite de memória do contêiner para $M$ unidades, de acordo com a requisição do cliente. Duas situações em relação à quantidade de memória podem ocorrer: (a) superestimação: a aplicação precisa de bem menos que $M$ de memória, levando a subutilização dos recursos; (b) subestimação: a aplicação precisa mais do que $M$ de memória, levando a uma queda de desempenho da execução ou mesmo a interrupção da mesma[Kubernetes 2020].

Para monitorar o uso de memória, o VEMoC coleta informações diretamente do cgroups. Já o Docker, além de dados do cgroups, coleta um conjunto de informações que podem não ser relevantes para o problema em questão. Não somente é importante avaliar a sobrecarga associada à coleta, mas também a frequência de atualização. Avaliações iniciais mostram que a coleta do uso de memória de um contêiner usando cgroups é duas ordens de grandeza mais rápida do que usando o API do Docker.

\section{Análise dos limites de memória}

Para analisar o efeito do limite de memória na execução de aplicações em Docker contêineres, foram realizados experimentos considerando dois jobs memory bound sintéticos, denotados por $J 1$ e $J 2$. Cada um dos jobs acessa $N$ elementos de um vetor. Enquanto $J 1$ acessa todos os elementos em sequência em $I$ iterações, no job $J 2$, o vetor é dividido em $m$ blocos tal que os elementos de cada bloco são acessados em sequência $I$ vezes antes de passar para o próximo bloco.

Os experimentos consideraram um vetor de $4096 \mathrm{MB}$, que é percorrido $I=4$ vezes e, no caso do $J 2, m=4$ blocos de 1024MB. O objetivo dos testes é avaliar o comportamento de ambas as aplicações quando executadas sob limites $M$ de memória 
variados. A versão do Docker utilizada foi a 19.03.13, com contêiner com imagem base do CentOS:7, em um máquina física com processador AMD FX(tm)-8300, 8 cores em 3.3GHz, 8GB de RAM, 1666MHz DDR3 e 12GB de swap.

A Figura 1 mostra a média dos tempos de dez execuções de cada um dos jobs, com $M=512$ até $4096 \mathrm{MB}$ com incrementos de $512 \mathrm{MB}$. Foram adicionados as configurações de $M=1040$ e $4110 \mathrm{MB}$ para uma análise mais detalhada. Os resultados mostram que, enquanto em aplicações como o $J 1$, uma má previsão da necessidade de memória pode causar uma grande degradação em seu desempenho devido principalmente ao uso de swap pelos contêineres, manipular o limite de memória em contêineres que executam jobs como J2 podem levar a uma melhor utilização da memória com uma sobrecarga mínima.

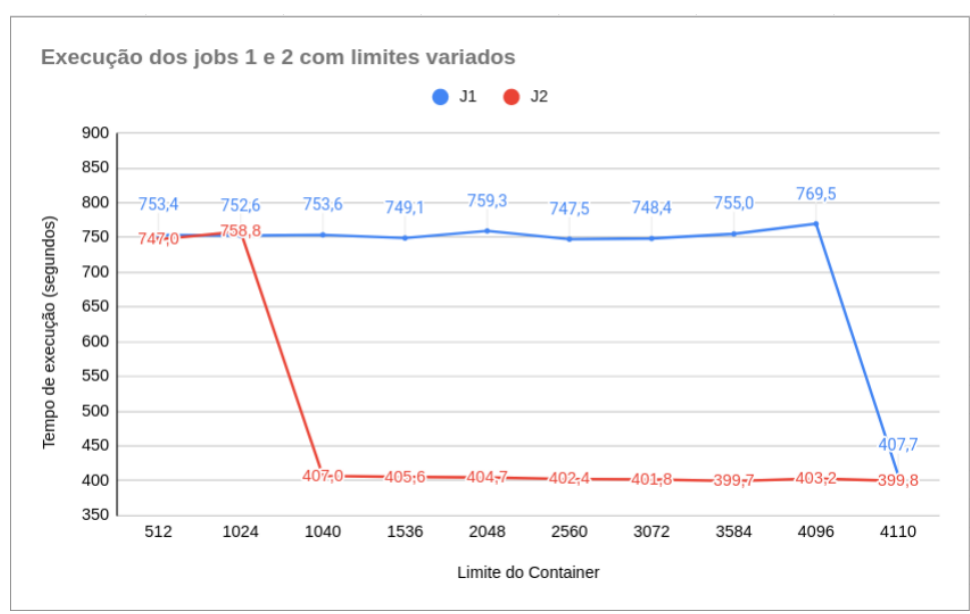

Figura 1. Execução de $J 1$ e $J 2$ variando limites de memória de seus contêineres.

\section{Conclusão e objetivos futuros}

A análise cuidadosa do uso de recursos em um ambiente virtual com Docker contêineres pode levar a um melhor custo-benefício na utilização de nuvens, tanto por parte dos usuários quanto dos provedores. Futuramente esse trabalho se desenvolverá para viabilizar a utilização de Docker contêineres no VEMoC, um gerenciador que explora elasticidade vertical em um ambiente com contêineres [Nicodemus et al. 2020], que atualmente é viável apenas para contêineres LXC. Isso será realizado através do aprofundamento dos desafios que o Docker traz na implementação da ferramenta e em como resolvê-los.

\section{Referências}

Canonical Ltd. (2020). Linux containers - lxc. https: / / linuxcontainers . org/ pt_br/lxc/introduction. Accessed on: 27/02/2020.

Docker Inc. (2020). Docker get started. https://docs.docker.com/ get-started/. Accessed on: 10/07/2020.

Kubernetes (2020). Kubernetes. https://kubernetes.io/. Accessed on: 01/07/2020.

Nicodemus, C., Boeres, C., and Rebello, V. E. F. (2020). Managing vertical memory elasticity in containers. In 2020 IEEE/ACM 13th International Conference on Utility and Cloud Computing (UCC), pages 1-10. 